\title{
Current Concepts in the Mandibular Condyle Fracture Management Part II: Open Reduction Versus Closed Reduction
}

\author{
Kang-Young Choi, Jung-Dug Yang, Ho-Yun Chung, Byung-Chae Cho \\ Department of Plastic and Reconstructive Surgery, Kyungpook National University School of Medicine, Daegu, Korea
}

\begin{abstract}
In the treatment of mandibular condyle fracture, conservative treatment using closed reduction or surgical treatment using open reduction can be used. Management of mandibular condylar fractures remains a source of ongoing controversy in oral and maxillofacial trauma. For each type of condylar fracture, the treatment method must be chosen taking into consideration the presence of teeth, fracture height, patient'sadaptation, patient's masticatory system, disturbance of occlusal function, and deviation of the mandible. In the past, closed reduction with concomitant active physical therapy conducted after intermaxillary fixation during the recovery period had been mainly used, but in recent years, open treatment of condylar fractures with rigid internal fixation has become more common. The objective of this review was to evaluate the main variables that determine the choice of an open or closed method for treatment of condylar fractures, identifying their indications, advantages, and disadvantages, and to appraise the current evidence regarding the effectiveness of interventions that are used in the management of fractures of the mandibular condyle.
\end{abstract}

Keywords Jaw fixation techniques / Mandibular condyle / Mandibular fractures / Motion therapy, continuous passive
Correspondence: Kang-Young Choi Department of Plastic and Reconstructive Surgery, Kyungpook National University School of

Medicine, 130 Dongduk-ro, Jung-gu, Daegu 700-721, Korea

Tel: $+82-53-420-5685$

Fax: +82-53-425-3879

E-mail:kychoi@knu.ac.kr

This article was invited as part of a panel presentation, which was one of the most highly rated sessions by participants, at the 69th Congress of the Korean Society of Plastic and Reconstructive Surgeons on November 11, 2011 in Seoul, Korea.

No potential conflict of interest relevant to this article was reported.

Received: 8 July 2012 • Revised: 9 July 2012 • Accepted: 10 July 2012

pISSN: 2234-6163 • elSSN: 2234-6171 • http://dx.doi.org/10.5999/aps.2012.39.4.301・ Arch Plast Surg 2012;39:301-308

\section{INTRODUCTION}

In the past, closed reduction with concomitant active physical therapy that is conducted after intermaxillary fixation during recovery period had been mainly used. However, as it has disadvantages such as metastasis of the fractured bone by muscle strength, abnormal occlusion due to inappropriate fixation, and inappropriate function of the temporomandibular joint (TMJ) due to disuse muscular atrophy caused by long-term intermaxillary fixation, open reduction has recently drawn attention. In particular, condyle fracture is satisfactorily treated by closed re- duction. Many researchers recommended closed reduction because of problems of surgical approach, such as infection, injury of nerve and blood vessel, and scar formation [1-3]. However, compared to previous open reduction, it has been currently more widely used by minimizing complications such as TMJ pain and arthritis, and mouth opening limitation via accurate reduction of bony fragment with the development of surgical instruments and surgical approaches.

However, it is still controversial over the selection of either closed or open reduction to treat condyle fracture depending on displacement severity and fracture site. Klotch and Lundy [4] 
and Widmark et al. [5] reported that open reduction should be conducted if fractured mandibular condyle is severely displaced, and that closed reduction may be conducted considering various factors such as elderly or pediatric patients, difficulty in the conduct of open reduction under systemic anesthesia, no other facial fracture, and secured stability of occlusion.

Haug and Assael [6] reported that no statistically significant difference in occlusion status and complication such as mandibular movement restriction was found between open and closed reductions for mandibular condyle fracture. Ellis et al. [7] reported that complications such as intraoperative bleeding and postoperative infection, facial nerve paralysis, functional disorder of the auriculotemporal nerve, and condyle growth disorder significantly increased when open reduction was conducted to treat condylar head and neck fractures, and that closed reduction was more advantageous than open reduction. Meanwhile, Brown and Jones [8] conducted rigid fixation using mini plate, reporting that no intermaxillary fixation was required. Tu and Tenhulzen [9]. Reported that fracture fixation using mini plate and screw shortened intermaxillary fixation period and prevented the disuse atrophy of the masticatory muscle, thereby achieving early opening, and that postoperative complications significantly decreased. Jeter et al. [10] reported that relatively satisfactory outcomes were obtained from closed reduction for condyle fracture, but that this method could cause mouth opening disorder, mandibular setback, temporomandibular pain, and functional disorders after long period after injured. They recommended that fracture reconstruction and rigid fixation via open reduction should be conducted on patients with condyle fracture to achieve immediate mouth opening movement, and that maintaining of intraoral hygiene, improvement of nutritional improvement, and normal pronunciation should be performed.

\section{TREATMENT OF MANDIBULAR CONDYLE FRACTURE ORIF VS. CRIMF}

\section{Closed reduction and functional therapy Method}

For closed reduction, intermaxillary fixation is conducted using arch bar and wire, followed by maintaining of the fixation of the maxilla and mandible for 2 to 4 weeks. After achieving stable union of the factored site, a wire for intermaxillary fixation is removed. Then, normal occlusion is induced after fixation using rubber, and soft diet is maintained for 2 weeks. Functional therapy that consists of passive mandibular movement exercise and mouth opening exercise is conducted and then clinical outcomes are observed. For mouth opening exercise, the physician holds the molar and mandibular border of the fracture side after standing behind the patient, and induces normal occlusion and normal mandibular movement by traction to the anterior inner inferior several times. At the same time, the patient opens his/her mouse for him/herself, and applies counter-force using hands to avoid mandibular deviation. Initial intermaxillary fixation period varies depending on literatures. The authors conduct initial intermaxillary fixation in intracapsular fracture patients aged less than 5 years for 2 weeks, in those aged 5 years or higher for 4 weeks, and in extracapsular fracture patients aged less than 8 years for 2 weeks.

\section{Advantage}

Closed reduction with functional therapy is a relatively safe treatment. No injury of nerves and blood vessels occur during the treatment, and no postoperative complications such as infection or scar occurs. In particular, complications such as fracture, loss, and eruption delay of the growing teeth can be avoided in pediatric patients as no tooth germ injury occurs because of no establishment of the crown of the permanent teeth [11] (Figs. $1,2)$.

\section{Disadvantage}

Long-term intermaxillary fixation has disadvantages of the injury of the periodontal tissue and buccal mucosa, poor oral hygiene, pronunciation disorder, imbalanced nutrition, mouth opening disorder, and respiration disorder $[12,13]$. In the case of conservative treatment using closed reduction, the growth disorder and excessive growth of the injured mandible may occur due to inappropriate reduction of bone fragments $[14,15]$ and the right and left displacement of the mandibular ramus or mandibular deviation upon opening may occur after conservative treatment [16] (Fig. 3). Many studies reported that facial asymmetry or TMJ disease may occur in pediatric patients aged 10 to 15 years due to growth disorder or functional disorder, and that in particular, the growth and functional disorders of the TMJ may occur in $20 \%$ to $25 \%$ of pediatric patients aged 7 to 10 years $[17,18]$.

\section{Open reduction}

Method

There are various operation methods of open reduction for madibular condyle fracture depending on fracture site and degree of bone fragment displacement. In general, they include preauricular approach, postauricular approach, submandibular approch, Risdon approach, combined approach, and retromandibular approach. Treatment type should be selected considering patient's age, preference, fracture type, fracture of other sites, and teeth status. 


\section{Fig. 1. An 18-year-old man with condyle head fracture due to traffic accident}

(A) Preoperative coronal view of 3D head computed tomography (CT). (B) Coronal view of 3D head CT after functional treatment for 4 weeks.
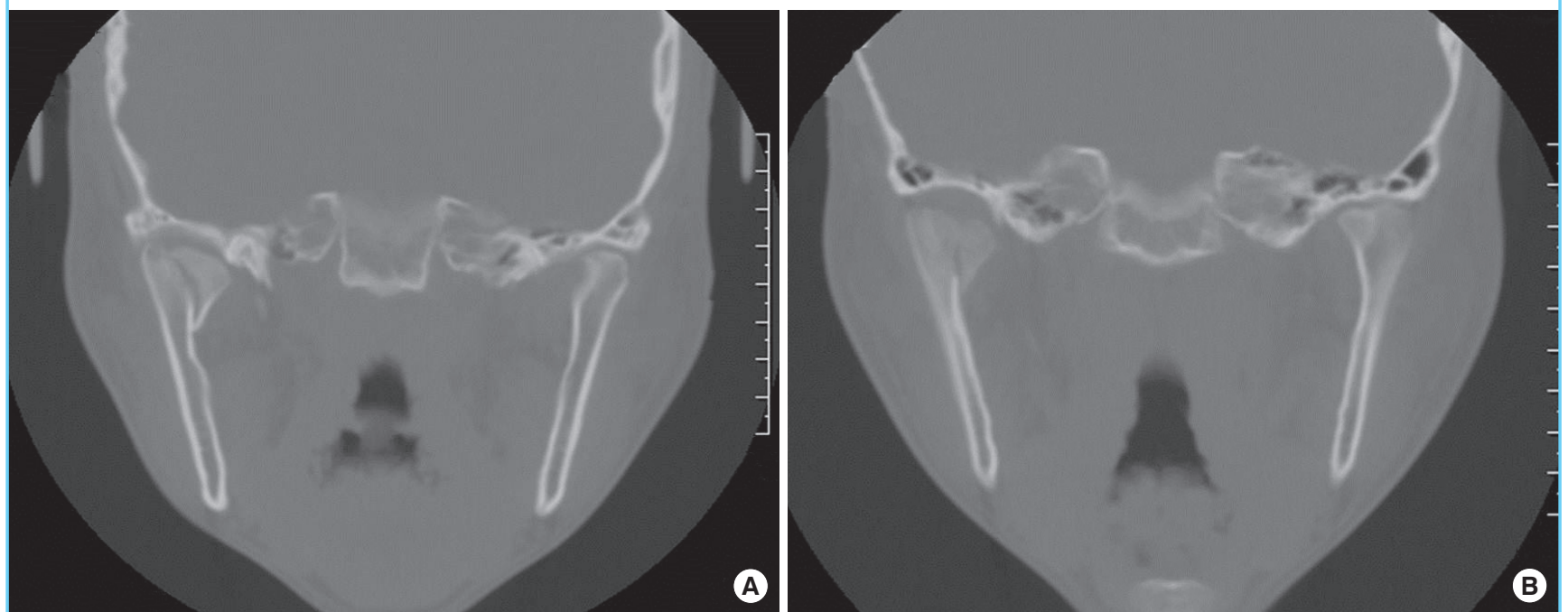

\section{Fig. 2. An 8-year-old girl with condyle head fracture due to slip down}

Serial 3D head computed tomography (CT) was checked during closed reduction and functional therapy. (A) Preoperative axial view of 3D head CT. (B) Axial view of 3D head CT after functional treatment for 2 months. (C) Axial view of 3D head CT after functional treatment for 4 months. (D) Preoperative Coronal view of 3D head CT. (E) Coronal view of 3D head CT after functional treatment for 2 months. (F) Coronal view of 3D head CT after functional treatment for 4 months.
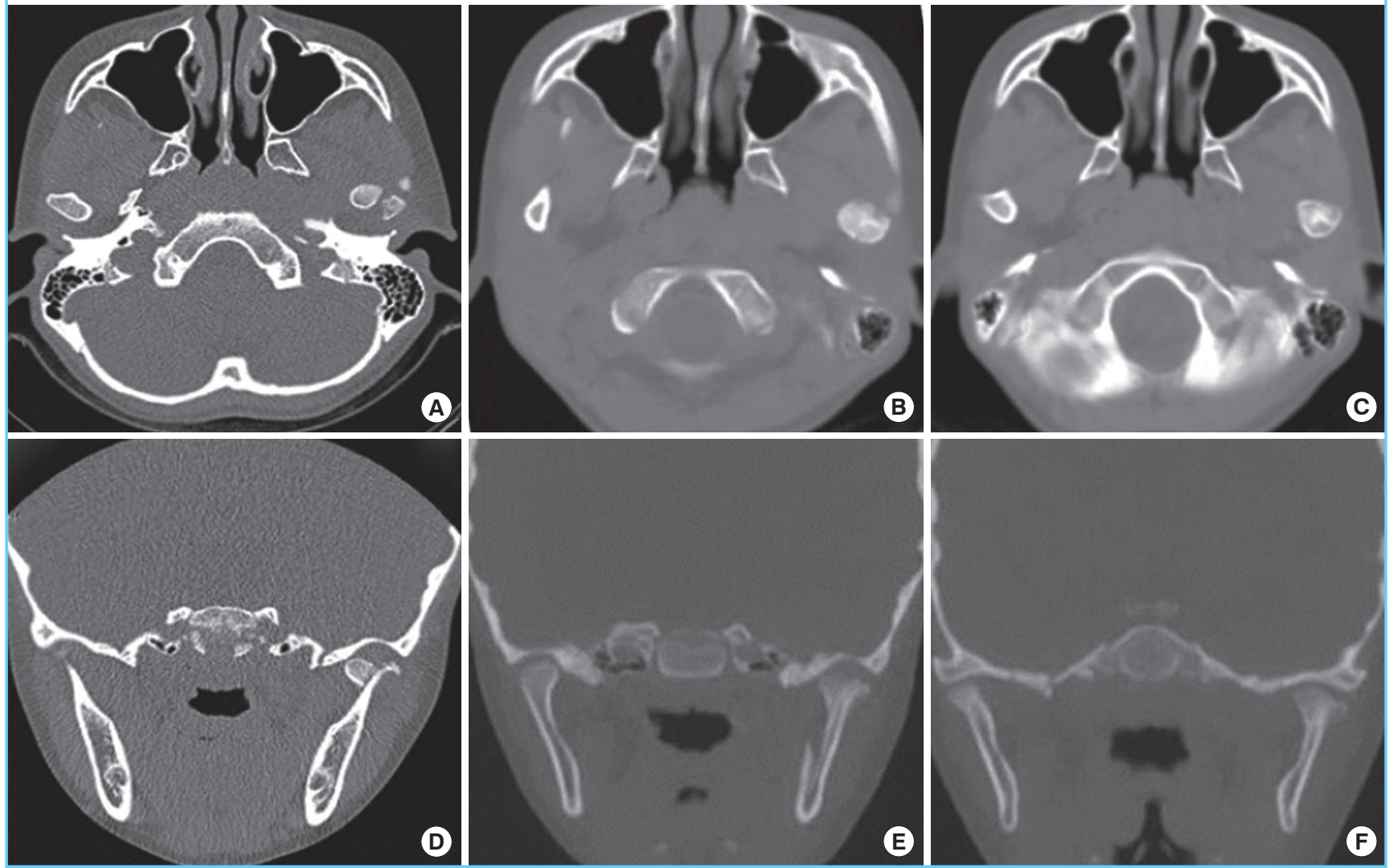

Advantage

Open reduction has advantages of the reduction of the displaced bony fragment to the most ideal anatomical site by a direct approach to the facture site. In addition, it can prevent complica- 
Fig. 3. A 39 years old woman with both condyle fracture due to traffic accident

(A) Intermaxillary fixation (IMF) with arch bar apply. (B) IMF and arch bar removal was done at postoperative 1 month. (C) After 2 years, the panorama plain film was checked. Functional treatment was applied initially for 2 months.
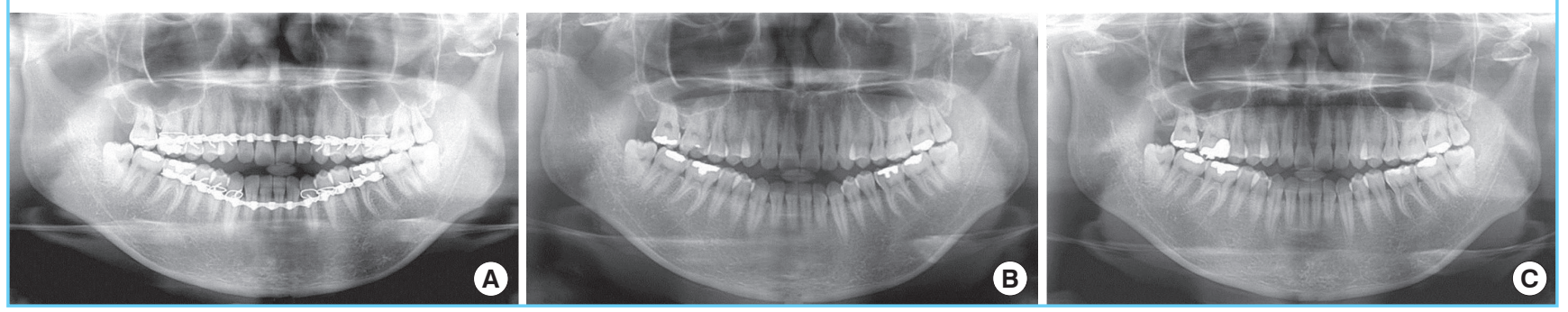

Fig. 4. A 70 years old woman with subcondylar fracture due to slip down

(A) Preoperative intermaxillary fixation with arch bar apply. (B) Intraoperative finding. (C) Panorama plain film was checked at postoperative 6 months. The subcondylar fracture was corrected by open reductionand internal fixation using pre-auricular approach.
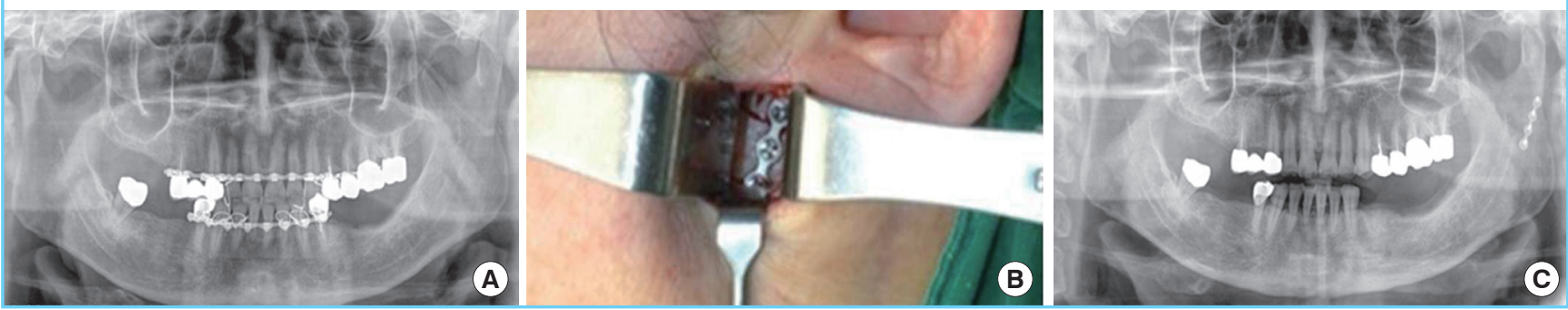

tions such as respiration disorder, pronunciation disorder, and severe nutritional imbalance by shortening intermaxillary fixation period via rigid fixation.

\section{Disadvantage}

Open reduction is an invasive treatment, which may cause injury of nerves or blood vessels during operation, and postoperative complications including infection. In addition, it has permanent scar though the surgery is conducted after designing the incision line considering aesthesis.

\section{Operative procedure}

\section{Preauricular approach}

Preauricular approach reduces condyle fracture by incising 3 to $4 \mathrm{~cm}$ from the inferior border of the tragus toward external auditory canal along the skin crease of the anterior part of the external ear (Fig. 4). It provides an easier approach to high condylar fracture such as intercapsular fracture, easy reduction of the injured soft tissues of the TMJ, and reduction via a direct inspection of the appropriate relationship among the condyle, disc, and joint with eyes. In particular, preauricular approach is very useful for the case of the condyle fragment anteromedially displaced by the pulling of the medial pterygoid [19]. However, an approach to the mandibular angle fracture is very difficult if the mandible should be pulled inferiorly to find the displaced proximal seg- ment. Furthermore, as the amount of exposing the mandibular ramus is very limited, rigid fixation using mini-plate is hard to be conducted if fracture site is positioned inferiorly to the mandibular condyle neck.

\section{Postauricular approach}

The postauricular approach is a method that reducesthe condyle fracture by incising from a site $3 \mathrm{~mm}$ posterior to the postauricular curved region along the curved region, and by incising the mastoid process inferiorly and the upper ear-attached region superiorly. It can be used for the reduction of high condyle fracture. This method has advantages of excellent aesthesis due to the approach from the posterior side of the ear, avoiding injuries ofthe facial nerve branch and superficial temporal artery, low risk of parotid injury, and securing the surgical field for the TMJ region. Meanwhile, it has disadvantages of a narrow surgical field for mandibular condyle neck fracture, difficulty in using surgical devices, complications such as external auditory canal stenosis, tinnitus, infection and necrosis of auricular cartilage, permanent auricular paresthesia due to injury of the external auditory canal, and longer wound closure time compared to the preauricular approach.

\section{Submandibular approach}

The submandibular approach reduces condyle fracture by con- 
ducting incision from a site 2 to $3 \mathrm{~cm}$ inferior to the mandibular inferior border, parallelly to the mandibular inferior border or along with the skin crease. Due to its easier approach to the mandibular ramus, inferior mandibular condyle, and coronoid notch, it is commonly used for mandibular condyle fracture. However, it has disadvantages of requiring excessive traction for reducing mandibular condyle fracture, requiring rigid fixation using percutaneous trocar for reducing high condyle fracture due to difficulty in an direct approach to the site of the fracture line formed, and requiring deep tunneling for mandibular condyle fracture due to a long distance from the incision line to the fixation site, and requiring the use of mini-plate due to poorly secured surgery field. Furthermore, it has disadvantages of the possible risk of the injury of inferior alveolar branch of the facial nerve, submandibular scar formation, difficulty in approaching the high condyle fracture site, and difficulty in examining the internal structure of the TMJ.

\section{Risdon approach}

Risdon approach is a method similar to submandibular approach. It can easily approach to the inferior region, ramus, gonial angle and posterior body of the mandibular condyle. If the upper flap is intensively retracted, even mandibular condyle inferior and neck fractures can be exposed. Reduction of bone fragments can be easily conducted by traction the mandibular gonial angle inferiorly. Meanwhile, like submandibular approach, Risdon approach requires excessive traction for high condyle fracture [20].

\section{Combined approach}

This method reduces both inferior and superior fractures of the mandibular condyle by applying preauricular approach and submandibular approach simultaneously. This method is very useful as mandibular subcondyle fracture is reduced using submandibular approach, and the superior fractures of the TMJ or mandibular condyle neck is approached via preauricular approach and bone fragments are reduced while putting in traction the mandible inferiorly. Meanwhile, due to the use of two approaches, combined approach has disadvantages of relatively longer operation time, large scar formation, high risk of the injury of facial nerve, and risk of secondary TMJ disease due to scar formation on the TMJ capsule by preauricular approach.

\section{Intraoral approach}

Intraoral approach reaches the mandibular condyle in a way similar to vertical ramus osteotomy. The incision line is formed along the anterior mandibular ramus and buccal sulcus. For the achievement of surgery field and device approach, the temporalis muscle attached to the mandibular ramus and the periosteum of the buccinator located at the body should be completely dissected to elevate them. This method has advantages of no scar formation and the minimum injury of facial nerves. Meanwhile, an approach using devices is difficult though operation field is secured using an endoscope. Furthermore, percutaneous trocar should be used for rigid fixation using metal plate after reduction. It has disadvantages of difficulties in the maintaining of bone fragment stability and in the observation of the internal structure of the TMJ for mandibular subcondyle fracture [10].

\section{Retromandibualar approach}

Retromandibualar approach reduces condyle fracture by dissecting the skin and subcutaneous tissue vertically to the mandibular angle using the $3-\mathrm{cm}$ incision line to the $5 \mathrm{~mm}$ inferior to the auricular lobe. This method provides easy reduction and rigid fixation for mandibular subcondyle fracture. Percutaneous trocar is not required as the method can tract the tissues anteriorly and superiorly at the sigmoid notch. It also provides reduction and rigid fixation for high condyle fracture, where incision length is small. Furthermore, this method has advantages of insignificant scar formation due to the incision made at the posterior mandibular ramus, and the sufficient exposure of bone fragments to the upper part of the mandibular ramus. However, it has disadvantages of risk of the injury of facial nerves and bleeding caused by the injury of blood vessels [20,21].

\section{Final check point after open reduction}

For the reduction of facial bone fracture, the authors suggest that after reducing other elements according to centric occlu condyle (CO) with normal functions should be identified, followed by performing the condyle fracture reduction. The sequence of reduction, however, is somewhat controversial. If reduction is conducted according to the fracture line and condyle type prior to other occlusion-related fracture, the result of subsequent reduction to other elements according to $\mathrm{CO}$ may cause occlusion interference during the normal movement of the mandible. In that case, trauma from occlusion (TFO) eventually occurs, thereby causing problems in the oral and maxillofacial system.

After facial bone surgery related to occlusion, disorders of functional movement that might occur later should be checked. In fact, as it is difficult to observe normal movement of the mandible and occlusal interference under systemic anesthesia, it is difficult to assess the aforementioned disorders. This is done by checking the disorder of mandibular functional movement after reduction according to $\mathrm{CO}$. when mouth opening is performed by holding the mandible with hands, translation movement occurs after appropriate rotation movement. At that point, if condyle head movement is palpitated at the preauricular area, man- 
dibular movement at the sagittal plane is considered good. No deflection of mandibular movement should occur at this point. Next, guidance teeth are identified from anterior and lateral movement after checking the attrition status of the occlusion surface. If the guidance teeth found during mandibular movement by maintaining the contact of the maxillary and mandibular teeth by holding the mandible with hands, and the guidance teeth during movement are identical, and if no premature contact occur in other teeth, functional movement is expected to be normal.

\section{GUIDELINE OF TREATMENT}

Mandibular condyle fracture is the most common fracture among mandibular fractures, the treatment methods for mandibular condyle fracture have been controversial. Since Zide and Kent [1] reported the relative and absolute indications of mandibular condyle fracture in 1983, open reduction via surgery has become controversial, and the new approaches of surgical reduction and fixation have been introduced and developed.

In particular, mandibular condyle fracture occurs by various causative factors, and has various treatment methods depending on the fracture location, patient's age, and fracture type. However, regardless ofthe treatment option, the purpose of the treatment of mandibular condyle fracture is to recover normal TMJ function via the reconstruction of appropriate anatomical position. Thus, assessment of treatment success, as well as the outcomes of an early treatment, should be conducted based on complications such as TMJ derangement, ankylosis of TMJ, or growth disorder via long-term follow-up. Therefore, it is important to control functional complications and aesthetic problems from a long-term perspective. The final goal of the treatment lies in the achievement of occlusal stability, normal mouth opening, normal TMJ movement, prevention of temporomandibular

\begin{tabular}{|l|}
\hline Table 1. Zide and Kent's indications for open reduction (1983) \\
\hline Absolute Indicaton \\
\hline Displacement into middle cranial fossa \\
Impossibility of obtaining adequate occlusion by closed reduction \\
Lateral extracapsular displacement \\
Invasion by foreign body \\
\hline Relative Indication \\
\hline Bilateral condylar fractures in an edentulous patient without a splint \\
Unilateral or bilateral condylar fractures where splinting cannot be accomplished \\
for medical reasons or because physiotherapy is Impossible \\
Bilateral condylar fractures with communited midfacial fractures, prognathisim or \\
retroprognathism \\
Periodontal problems \\
Loss of teeth \\
Unilateral condylar fracture with unstable base \\
\hline From Zide and Kent, with permission from Elsevier [1].
\end{tabular}

joint derangement and joint pain, and prevention of growth disorder in patients with mandibular fracture by selecting an appropriate treatment method between closed and open reductions.

\section{Zide and Kent's indication of open reduction (1983)}

For indications of open reduction on mandibular condyle fracture, Zide and Kent [1] suggested that absolute indications should include displacement into middle cranial fossa, inappropriate occlusal restoration by closed reduction, lateral extracapsular displacement, and foreign material of the fracture site, and that relative indications should include bilateral mandibular condyle fracture of edentulous patients who can not have splint, impossible intermaxillary fixation and physical therapy due to internal diseases, bilateral mandibular condyle fracture with comminuted fracture of other facial bone, and bilateral mandibular condyle fracture with jaw deformities (Table 1). They also suggested that factors involved in the selection of open reduction include the location of the displaced mandibular condyle, fracture site, time delayed after fracture, patient's individual characteristics, edema severity, selection of incision line, and fixation type.

\section{Mathes (1983)}

Klotch and Lundy [4] and Choi et al. [22-24] suggested that angulation between the fractured fragments in excess of 30 degrees and fracture gap between the bone ends exceeding 4 or $5 \mathrm{~mm}$, lateral override, and lack of contact of the fractured fragments should be considered before justifying open reduction (Table 2) [25].

\section{Table 2. Treatment protocol (Mathes)}

\begin{tabular}{|l|}
\hline Open Reduction Indication \\
\hline Malocclusion with CR \\
Fragment angulation: more $30^{\circ}$ \\
Bone gap: more $4-5 \mathrm{~mm}$ \\
Lateral override \\
Lack of contact of the fracture fragment \\
\hline Preferred for Open Reduction \\
\hline Any low, dislocated subcondylar fracture \\
Low condylar fracture with multiple fractured mandible or maxillary or Le Fort \\
fracture \\
Low condylar fracture with displacement of condylar head out of the glenoid fossa \\
Condylar fragment $14^{\circ}$ - medial tilt \\
Ramus shortening - 5\% \\
Bilateral fracture with open bite \\
Gross fracture end malalignment \\
Fracture - dislocation \\
Abnormal function, malocclusion \\
\hline From Mathes and Hentz, with permission from Elsevier [24]. \\
\hline
\end{tabular}


Table 3. AAOMS special committee on parameters of care indications for open reduction (2003)

Physical evidence of fracture

Imaging evidence of fracture

Malocclusion

Mandibular dysfuncton

Abnormal relationship of jaw

Presence of foreign bodies

Lacerations and/or hemorrhage in external auditory canal

Hemotympanum

Cerebrospinal fluid otorrhea

Effusion

Hamarthrosis

Permission from American Association of Oral and Maxillofacial Surgeons [26]

\section{AAOMS (2003)}

In 2003, American Association of Oral and Maxillofacial Surgery suggested an international guideline on the treatment of mandibular condyle fracture. According to the guideline, open reduction is recommended for the cases of mandibular condyle fracture suspected in clinical and radiologic examinations to prevent complications such as functional or growth disorders (Table 3) [26].

\section{Author's method}

Researchers supporting open reduction on mandibular condyle fracture report that the anatomically and functionally accurate reconstruction of bone fragments is important. Despite the good outcomes of conservative treatment, mouth opening deflection and chronic dull pain may occur during a long-term follow-up. Thus, open reduction is recommended for the cases of indication of open reduction, difficulty in the appropriate treatment of fracture via closed reduction, and high risk of complication after mandibular condyle fracture (Fig. 5).

For the treatment of mandibular condyle fracture in pediatric patients, anatomical structure and physiological and psychiatric development should be considered as they differ between pediatric and adult patients though pathogenesis and clinical manifestation are similar between the two groups. Pediatric patients have the facial bone covered with thick soft tissues, elastic bone structure, and the thin cortical bone and also have a large amount of premature trabecular bone. Thus, no severe impact occurs upon receiving trauma. In general, incomplete fracture with a greenstick type occurs [27].

As teeth alignment has a status of primary or mixed dentition, and most of the growing crown of the permanent tooth has not been completely established yet, the ratio of bone tissue to the tooth is relatively low. Thus, un-erupted teeth are easily included in the fracture line, and complications such as the fracture, loss,

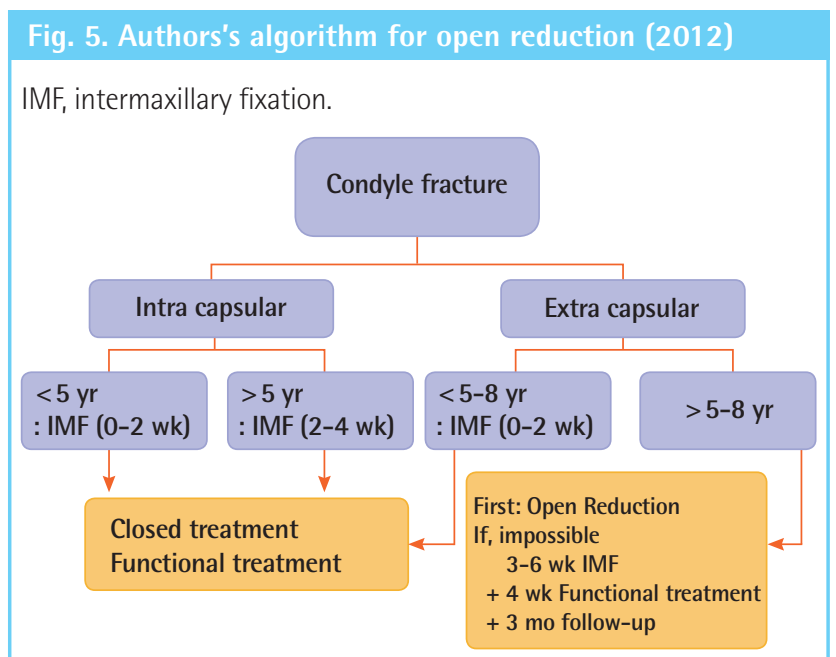

delayed eruption of the growing tooth may occur if unerupted permanent teeth are injured. If the intermaxillary fixation period is maintained for 2 weeks or more, mandibular growth disorder and the injury of the teeth undergoing eruption may occur. Thus, in pediatric patient, open reduction of the condyle is invasive for itself, and has a risk of facial nerve injury. Furthermore, no significant difference in prognosis is found compared to closed reduction. Thus, a non-surgical approach is recommended, in which intermaxillary fixation period should be 2 weeks or less.

Accordingly, the authors principally conduct functional therapy after closed reduction for intracapsular fracture in the treatment of mandibular condyle fracture. Intermaxillary fixation is maintained for 2 weeks in patients aged less than 5 years, and for 2 to 4 weeks in patients aged 5 years or higher depending on physical development and patient compliance. In the case of extracapsular mandibular condyle fracture, closed reduction is conduced, followed by functional therapy after 2-week intermaxillary fixation in patients aged 5 to 8 years. Meanwhile, open reduction is preferentially considered in patients aged 8 years or higher. If open reduction is impossible, closed reduction is conducted, followed by 4-week functional therapy after 3 to 6-week intermaxillary fixation. Then, occlusion and TMJ dysfunction are carefully observed every 3 months (Fig. 5).

\section{CONCLUSIONS}

In the treatment of mandibular condyle fracture, conservative treatment using closed reduction and surgical treatment using open reduction are used. However, it is still controversial over indications. Thus, treatment type should be selected considering patient's age, fracture type, patient's systemic status, other fracture, teeth, and possibility of occlusal restoration by inter- 
maxillary fixation, and existence of foreign materials. In the final determination of treatment plan, the advantage, disadvantage, and risk of each treatment, and risk of complications should be sufficiently discussed with patients and patient's guardians. In addition, the treatment plan of mandibular condyle fracture should be established considering the aforementioned various factors rather than the criteria for absolute indications using the treatment guideline suggested by the authors.

\section{REFERENCES}

1. Zide MF, Kent JN. Indications for open reduction of mandibular condyle fractures. J Oral Maxillofac Surg 1983;41: 89-98.

2. Goss AN, Bosanquet AG. The arthroscopic appearance of acute temporomandibular joint trauma. J Oral Maxillofac Surg 1990;48:780-3.

3. Wood GD. Assessment of function following fracture of the mandible. Br Dent J 1980;149:137-41.

4. Klotch DW, Lundy LB. Condylar neck fractures of the mandible. Otolaryngol Clin North Am 1991;24:181-94.

5. Widmark G, Bagenholm T, Kahnberg KE, et al. Open reduction of subcondylar fractures: a study of functional rehabilitation. Int J Oral Maxillofac Surg 1996;25:107-11.

6. Haug RH, Assael LA. Outcomes of open versus closed treatment of mandibular subcondylar fractures. J Oral Maxillofac Surg 2001;59:370-5.

7. Ellis E 3rd, McFadden D, Simon P, et al. Surgical complications with open treatment of mandibular condylar process fractures. J Oral Maxillofac Surg 2000;58:950-8.

8. Brown JS, Jones K. Delayed miniplate osteosynthesis for mandibular fractures. Br J Oral Maxillofac Surg 1991;29: 286.

9. Tu HK, Tenhulzen D. Compression osteosynthesis of mandibular fractures: a retrospective study. J Oral Maxillofac Surg 1985;43:585-9.

10. Jeter TS, Van Sickels JE, Nishioka GJ. Intraoral open reduction with rigid internal fixation of mandibular subcondylar fractures. J Oral Maxillofac Surg 1988;46:1113-6.

11. Kaban LB, Troulis MJ. Pediatric oral and maxillofacial surgery. Philadelphia: Saunders; 2004.

12. Coletti DP, Salama A, Caccamese JF Jr. Application of intermaxillary fixation screws in maxillofacial trauma. J Oral Maxillofac Surg 2007;65:1746-50.
13. Gaujac C, Ceccheti MM, Yonezaki F, et al. Comparative analysis of 2 techniques of double-gloving protection during arch bar placement for intermaxillary fixation. J Oral Maxillofac Surg 2007;65:1922-5.

14. Khosla M, Boren W. Mandibular fractures in children and their management. J Oral Surg 1971;29:116-21.

15. Dahlstrom L, Kahnberg KE, Lindahl L. 15 years follow-up on condylar fractures. Int J Oral Maxillofac Surg 1989;18: 18-23.

16. Norholt SE, Krishnan V, Sindet-Pedersen S, et al. Pediatric condylar fractures: a long-term follow-up study of 55 patients. J Oral Maxillofac Surg 1993;51:1302-10.

17. Kaban LB. Diagnosis and treatment of fractures of the facial bones in children 1943-1993. J Oral Maxillofac Surg 1993;51:722-9.

18. Kahl B, Fischbach R, Gerlach KL. Temporomandibular joint morphology in children after treatment of condylar fractures with functional appliance therapy: a follow-up study us computed tomography. Dentomaxillofac Radiol 1995;24:37-45.

19. Chuong R, Piper MA. Open reduction of condylar fractions of the mandible in conjunction with repair of discal injury: a preliminary report. J Oral Maxillofac Surg 1988;46:257-63.

20. Ellis E 3rd, Dean J. Rigid fixation of mandibular condyle fractures. Oral Surg Oral Med Oral Pathol 1993;76:6-15.

21. Ellis E, Zide MF, Manson PN. Surgical approaches to the facial skeleton. Baltimore: Williams \& Wilkins; 1995.

22. Choi BH, Kim KN, Kim HJ, et al. Evaluation of condylar neck fracture plating techniques. J Craniomaxillofac Surg 1999;27:109-12.

23. Choi BH, Yoo JH. Open reduction of condylar neck fractures with exposure of the facial nerve. Oral Surg Oral Med Oral Pathol Oral Radiol Endod 1999;88:292-6.

24. Choi BH, Yi CK, Yoo JH. Clinical evaluation of 3 types of plate osteosynthesis for fixation of condylar neck fractures. J Oral Maxillofac Surg 2001;59:734-7.

25. Mathes SJ, Hentz VR. Plastic surgery. 2nd ed. Philadelphia: Saunders Elsevier; 2006.

26. American Association of Oral and Maxillofacial Surgeons [Internet]. Rosemont: American Association of Oral and Maxillofacial Surgeons; c2012 [cited 2012 Jul 7]. Avaiable from: http://http://www.aaoms.org/.

27. Guven O, Keskin A. Remodelling following condylar fractures in children. J Craniomaxillofac Surg 2001;29:232-7. 